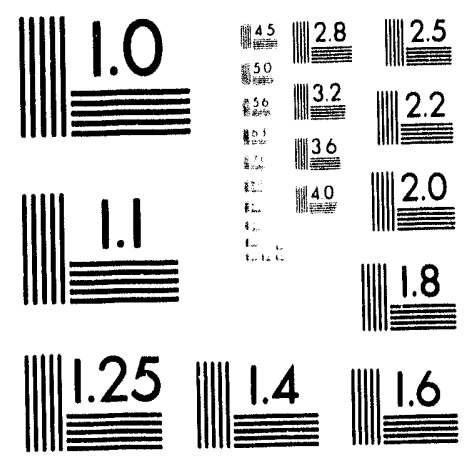



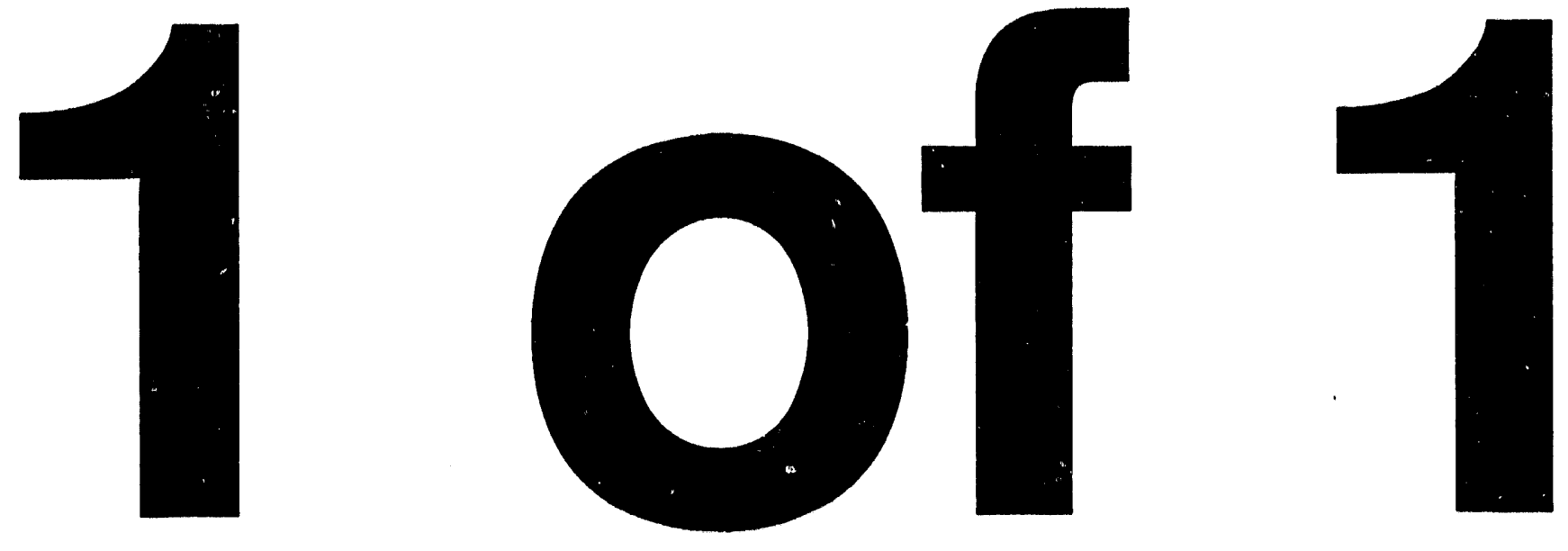
LA-UR. $93-3502$

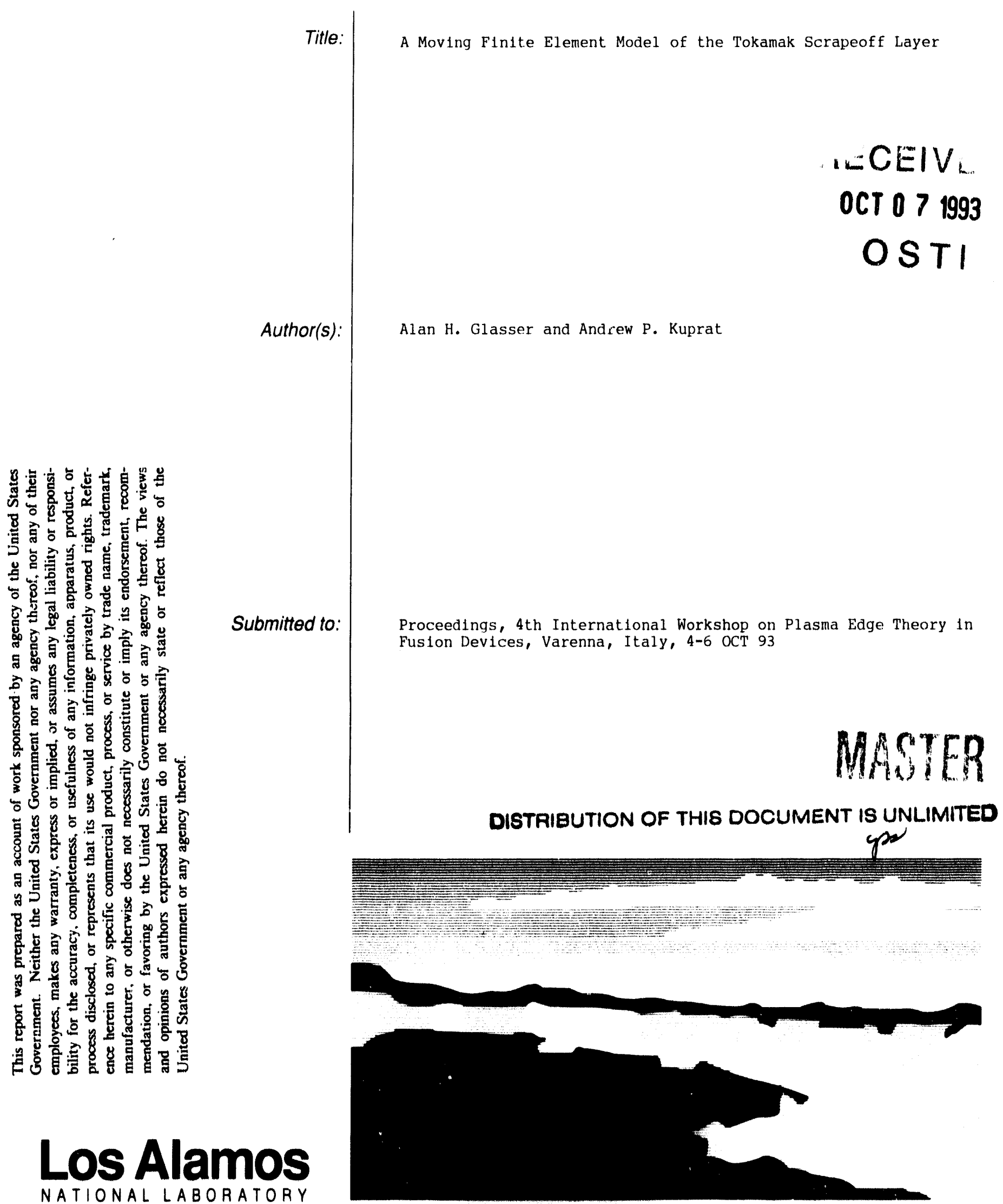

Submitted to:

Proceedings, 4th International Workshop on Plasma Edge Theory in usion Devices, Varenna, Italy, 4-6 OCT 93

TIONAL LABORATORT

Lus Alamos National Laboratory, an attirmative action/equal opportunity empldyer, is operated by the University of California for the U.S. Department of Energy under contract W-7405-ENG-36. By acceptance of thla anticle, the publisher recognizes that the U.S. Government retains a nonexclusive, royalty-free license to publish or reproduce the published form of this contribution, or to allow others to do so, for U.S. Government purposes. The Los Alamos National Laboratory requests that the publisher identify this article as work performed under the auspices of the U.S. Department of Energy. 


\title{
A Moving Finite Element Model of the Tokamak Scrapeoff Layer
}

\author{
Alan H. Glasser and Andrew P. Kuprat
}

Los Alamos National Laboratory

\begin{abstract}
Most numerical simulations of the tokamak scrapeoff layer use a mapping to flux coordinates and a piecewise equidistributed grid in those coordinates to resolve the multiple length scales and anisotropy characteristic of this problem. We have developed an alternative numerical method using simple cylindrical coordinates with a complex adaptive grid scheme. It is based on an unstructured grid of triangles which move adaptively, aligning themselves with the magnetic ficld and concentrating in regions of sharp gradients.
\end{abstract}

\section{Introduction}

Numerical simulation of the scrapeoff layer of a tokamak is challenging because of high anisotropy and multiple length and time scales. Most approaches are based on mapping the physical domain to a flux coordinate system based on a fixed equilibrium magnetic field. ${ }^{1}$ This approach suffers from some numerical difficulties. The flux coordinate system has a singularity at the $\mathrm{x}$-point, it cannot naturally adapt to regions of sharp gradients near the plates, and it cannot treat time-dependent magnetic fields. Some methods use orthogonal coordinates, which cannot easily accommodate divertor plates at oblique angles to the magnetic field.

We have developed an alternative approach, using the adaptive grid method of Moving Finite Elements (MFE). ${ }^{2}$ The computation is done in simple cylindrical coordinates $(R, Z)$. Instead of mapping to a flux coordinate system, a nonuniform, adaptive, unstructured grid of triangles is used to discretize the nonlinear, timedependent, partial differential equations of the fluid model with 2D linear finite elements. The vertices of the grid move smoothly and continuously, together with the time evolution of the solution, following the standard prescription of the method of MFE. The grid automatically adapts to the evolving solution, packing itself into regions of sharp gradients and aligning itself with the magnetic ficld in regions where 
the poloidal field is strong. A stiffly-stable, second-order accurate time step is used to treat multiple time scales. A technique called Graph Massage is used to occasionally create and annililate nodes, enabling our unstructured mesh to avoid tangling and to resolve the solution in a manner superior to that possible with a moving mesh of fixed topology. The data structures and techniques of Graph Massage have also made it straightforward to accomplish the related task of initially gridding the non-simply connected computational domain.

The rest of this paper is organized as follows. Section 2 presents our physical model; Section 3 describes our numerical methods; Section 4 presents results; and Section 5 discusses our conclusions.

\section{Physical Models}

Our physical model is determined by two goals. First, we want to verify the ability of our method to handle the numerical challenges presented by edge plasma modeling, including anisotropy, local regions of large gradients, and multiple time scales. Second, we want to develop a full, realistic edge code capable of competing with other numerical methods in this field. Our initial effort involved a simple model heat equation, with anisotropic and nonlinear thermal conductivity; this work has reached a satisfactory conclusion. Our present work treats the much more complete system of Braginskii's fluid equations for electrons and one species of ions. ${ }^{3}$ This code has been written and will be described here, but it has not yet been successfully run. Therefore, the results presented in Section 4, illustrating the method, are from the previous code. We discuss both of these models here. Future work will incorporate neutrals, impurities, and radiation.

Our simple model anistropic tokamak heat equation is

$$
\frac{\partial T}{\partial t}=\nabla \cdot(\chi \cdot \nabla T), \quad \chi=\chi_{\|} \hat{\mathbf{e}}_{1} \hat{\mathbf{e}}_{1}+\chi_{\perp}\left(\mathbf{I}-\hat{\mathbf{e}}_{1} \hat{\mathbf{e}}_{1}\right), \quad \hat{\mathbf{e}}_{1} \equiv \mathbf{B} / B
$$

The thermal conductivity tensor $\chi$ is defined in terms of the unit vector $\hat{\mathbf{e}}_{1}$ along $\mathbf{a}$ magnetic field B obtained from a numerical solution to the Grad-Shafranov equation. The parallel and transverse conductivities are taken to scale as

$$
\chi_{\|}=\chi_{0}\left(T / T_{0}\right)^{5 / 2}, \quad \chi_{\perp}=\chi_{\|} /\left[1+\left(T / T_{0}\right)^{3}\right],
$$

with $\chi_{0}=1$ and $T_{0}=.4$ in the example discussed in Section 4 . These scaling 
assumptions mimic the behavior of the classical transport coefficients and test the ability of the code to treat nonlinearity and anisotropy.

Our more complete and realistic model is based on Braginskii's equations. These include particle conservation, with $n=n_{e}=n_{i}$,

$$
\frac{\partial n}{\partial t}+\nabla \cdot(n \mathbf{v})=0
$$

conservation of momentum,

$$
\rho\left(\frac{\partial \mathbf{v}}{\partial t}+\mathbf{v} \cdot \nabla \mathbf{v}\right)=\frac{1}{c} \mathbf{j} \times \mathbf{B}-\nabla p-\nabla \cdot \pi,
$$

the heat equation for each species,

$$
\frac{3}{2} n\left(\frac{\partial T_{j}}{\partial t}+\mathbf{v}_{j} \cdot \nabla T_{j}\right)+n T_{j} \nabla \cdot \mathbf{v}_{j}+\nabla \cdot \mathbf{q}_{j}+\pi_{j}: \nabla \mathbf{v}_{j}=\frac{1}{n c} \mathbf{j} \cdot \mathbf{R},
$$

Ohm's law,

$$
n e\left(\mathbf{E}+\frac{1}{c} \mathbf{v} \times \mathbf{B}\right)=\mathbf{R}+\frac{1}{c} \mathbf{j} \times \mathbf{B}-\nabla p_{e},
$$

Maxwell's equations in the form

$$
\mathbf{E}=-\nabla \varphi-\frac{1}{c} \frac{\partial \mathbf{A}}{\partial t}, \quad \mathbf{B}=\nabla \times \mathbf{A}, \quad \nabla^{2} \mathbf{A}=-\frac{4 \pi}{c} \mathbf{j},
$$

and the quasineutrality equation

$$
\nabla \cdot \mathbf{j}=0
$$

The momentum exchange term is given by

$$
\begin{gathered}
\mathbf{R}=\mathbf{R}_{u}+\mathbf{R}_{T}, \\
\mathbf{R}_{u}=\frac{1}{n e} \alpha \cdot \mathbf{j}=\frac{1}{n e}\left[\alpha_{\|} \hat{\mathbf{e}}_{1} \hat{\mathbf{e}}_{1} \cdot \mathbf{j}-\alpha_{\wedge} \hat{\mathbf{e}}_{1} \times \mathbf{j}+\alpha_{\perp}\left(\mathbf{I}-\hat{\mathbf{e}}_{1} \hat{\mathbf{e}}_{1}\right) \cdot \mathbf{j}\right], \\
\mathbf{R}_{T}=-\beta \cdot \nabla T=-\left[\beta_{\|} \hat{\mathbf{e}}_{1} \hat{\mathbf{e}}_{1} \cdot \nabla T+\beta_{\wedge} \hat{\mathbf{e}}_{1} \times \nabla T+\beta_{\perp}\left(\mathbf{I}-\hat{\mathbf{e}}_{1} \hat{\mathbf{e}}_{1}\right) \cdot \nabla T\right],
\end{gathered}
$$

while the electron heat flux vector is given by

$$
\mathbf{q}=\mathbf{q}_{u}+\mathbf{q}_{T}
$$




$$
\begin{gathered}
\mathbf{q}_{u}=-\frac{T}{n e} \beta \cdot \mathbf{j}=-\frac{T}{n e}\left[\beta_{\|} \hat{\mathbf{e}}_{1} \hat{\mathbf{e}}_{1} \cdot \mathbf{j}+\beta_{\wedge} \hat{\mathbf{e}}_{1} \times \mathbf{j}+\beta_{\perp}\left(\mathbf{I}-\hat{\mathbf{e}}_{1} \hat{\mathbf{e}}_{1}\right) \cdot \mathbf{j}\right] \\
\mathbf{q}_{T}=-\chi \cdot \nabla T=-\left[\chi_{\|} \hat{\mathbf{e}}_{1} \hat{\mathbf{e}}_{1} \cdot \nabla T+\chi_{\wedge} \hat{\mathbf{e}}_{1} \times \nabla T+\chi_{\perp}\left(\mathbf{I}-\hat{\mathbf{e}}_{1} \hat{\mathbf{e}}_{1}\right) \cdot \nabla T\right]
\end{gathered}
$$

satisfying Onsager relations; the ion heat flux is given by $\mathrm{E}_{\mathrm{g}}$. (13) alone. For the viscous tensor, we retain only the terms arising from parallel and transverse gradients of the ion parallel velocity. All parallel transport coefficients are flux limited to treat local regions of long mean free path, and all transverse transport coefficients are cnhanced by anomalous contributions.

Our final system of 5 equations is obtained from Eqs. (3) - (14) as follows. Equations (3) and (5) are used to determine $n_{e}, T_{e}$, and $T_{i}$; the parallel component of $\mathrm{Eq}_{\mathrm{q}}$. (4) is used to determine the parallel momentum density $\pi_{\|}$; and Eq. (8) is used to determine the electrostatic potential $\varphi$. The transverse components of Eq. (6) are used to determine $v_{\perp}$, while the transverse components of Eq. (4) are used to determine $j_{\perp}$ and the parallel component of $\mathrm{E}_{1}$. (7) is used to determine $j_{\|}$. Our boundary conditions are as follows:

\section{Core Wall and Private Plates}

$\begin{array}{lll}n=3 \times 10^{19} \mathrm{~m}^{-3} & \left.\hat{\mathbf{n}} \cdot \nabla n\right|_{\text {out }}=\left.\hat{\mathbf{n}} \cdot \nabla n\right|_{\text {iu }} & \left.\hat{\mathbf{n}} \cdot \nabla n\right|_{\text {out }}=0 \\ T_{e}=250 \mathrm{eV} & \left.\hat{\mathbf{n}} \cdot \nabla T_{e}\right|_{\text {out }}=\left.\hat{\mathbf{n}} \cdot \nabla T_{e}\right|_{\text {in }} & q_{e} \|=\left(\delta_{e}-5 / 2\right) n T_{e} v_{e \|} \\ T_{i}=250 \mathrm{eV} & \left.\hat{\mathbf{n}} \cdot \nabla T_{i}\right|_{\text {out }}=\left.\hat{\mathbf{n}} \cdot \nabla T_{i}\right|_{\text {in }} & q_{i \|}=\left(\delta_{i}-5 / 2\right) n_{i} T_{i} v_{i} \| \\ p i_{\|}=0 & \left.\hat{\mathbf{n}} \cdot \nabla p i_{\|}\right|_{\text {out }}=\left.\hat{\mathbf{n}} \cdot \nabla p i_{\|}\right|_{\text {in }} & p i_{\|}=n\left[m_{i}\left(T_{e}+T_{i}\right)\right]^{1 / 2} \\ \left.\hat{\mathbf{n}} \cdot \nabla \varphi\right|_{\text {out }}=0 & \left.\hat{\mathbf{n}} \cdot \nabla \varphi\right|_{\text {out }}=\left.\hat{\mathbf{n}} \cdot \nabla \varphi\right|_{\text {in }} & \varphi=\kappa\left(T_{e}, T_{i}\right) T_{e} / e\end{array}$

where $\hat{\mathbf{n}}$ denotes the unit vector normal to the boundary.

\section{Moving Finite Elements}

Both models describe in Section 2 can be expressed in the general form

$$
\frac{\partial \mathbf{u}}{\partial t}+\nabla \cdot \mathbf{F}=\mathbf{S}
$$

where $t$ is the time, $\mathbf{x}$ is a vector of independent spatial varinbles, $\mathbf{u}$ is an $n$-vector of unknowns. For each component of $\mathbf{u}$ there is a flux $\mathbf{F}$ and a source $\mathbf{S}$ with the general form

$$
\mathbf{F}=\mathbf{C}(t, \mathbf{x}, \mathbf{u})-\mathbf{D}(t, \mathbf{x}, \mathbf{u}) \cdot \nabla \mathbf{u}, \quad \mathbf{S}=\mathbf{S}(t, \mathbf{x}, \mathbf{u}, \nabla \mathbf{u})
$$


where $\mathbf{C}$ represents convective flux and $\mathbf{D}$ is a diffusion tensor, and all functions may depend in an arbitrary nonlinear manner on their arguments. A variational for Eq. (15) is obtained by noting that

$$
L=\frac{1}{2} \int\left[\frac{\partial \mathbf{u}}{\partial t}+\nabla \cdot \mathbf{F}-\mathbf{S}\right]^{2} w(t, \mathbf{x}, \mathbf{u}, \nabla \mathbf{u}) d \mathbf{x},
$$

with $w$ an arbitrary weight function, is variational in the sense that

$$
\frac{\delta L}{\delta(\partial \mathbf{u} / \partial t)}=0
$$

recovers Eq. (15).

The general class of conventional Galerkin methods is obtained from Eqq. (17) and (18) by expanding $\mathbf{u}$ in a set of basis functions,

$$
\mathbf{u}(\mathbf{x}, t)=\mathbf{u}_{i}(t) \alpha_{i}(\mathbf{x})
$$

where the $\mathbf{u}_{i}(t)$ are time-dependent amplitudes and the $\alpha_{i}(\mathbf{x})$ are the spatiallyvarying basis functions, which may, for example, be Fourier series, orthogonal polynomials, or conventional fixed finite elements. Since the time derivative of $E_{\mathfrak{q}}$. (19) contains only the amplitude variations,

$$
\dot{\mathbf{u}}(\mathbf{x}, t)=\dot{\mathbf{u}}_{i}(t) \alpha_{i}(\mathbf{x}),
$$

the discretized equations of the method are obtained from

$$
\frac{\delta L}{\delta \dot{\mathbf{u}}_{i}}=0
$$

which yields a coupled system of ordinary differential equations,

$$
\left(\alpha_{i}, \alpha_{j}\right) \dot{\mathbf{u}}_{j}=\left(\alpha_{i}, \mathbf{g}\right),
$$

where $\mathbf{g}=\mathbf{S}-\nabla \cdot \mathbf{F}$.

The key issue for any adaptive grid method is how to move the grid. In Moving Finite Elements, this issue is resolved by treating the grid positions in exactly the same manner as the amplitudes, i.e. as variational parameters. For basis functions 
we choose linear finite elements on a grid of irregularly shaped and connected triangles in 2D, tetrahedra in 3D, and simplices in general. Because the grid is allowed to move, we replace Eqs. (19) and (20) by

$$
\begin{gathered}
\mathbf{u}(\mathbf{x}, t)=\mathbf{u}_{i}(t) \alpha_{i}\left[\mathbf{x}, \mathbf{s}_{j}(t)\right], \\
\dot{\mathbf{u}}(\mathbf{x}, t)=\dot{\mathbf{u}}_{i}(t) \alpha_{i}(\mathbf{x}, t)+\dot{\mathbf{s}}_{i}(t) \beta_{i}(\mathbf{x}, t),
\end{gathered}
$$

where $\beta_{i}$ is defined as the coefficient of $\dot{\mathbf{s}}_{i}$ obtained by differentiating Eq. (23). Then Eqs. (21) and (22) are generalized to

$$
\begin{gathered}
\frac{\delta L}{\delta \dot{\mathbf{u}}_{i}}=\frac{\delta L}{\delta \dot{\mathbf{s}}_{i}}=0, \\
\left(\alpha_{i}, \alpha_{j}\right) \dot{\mathbf{u}}_{j}+\left(\alpha_{i}, \beta_{j}\right) \dot{\mathbf{s}}_{j}=\left(\alpha_{i}, \mathbf{g}\right), \\
\left(\beta_{i}, \alpha_{j}\right) \dot{\mathbf{u}}_{j}+\left(\beta_{i}, \beta_{j}\right) \dot{\mathbf{s}}_{j}=\left(\beta_{i}, \mathbf{g}\right) .
\end{gathered}
$$

Just as Eq. (22) determines the amplitudes by minimizing the positive-definite variational over the space of amplitudes, Eqgs. (20) and (27) determine the amplitudes and node positions by minimizing over a larger space, and thus obtain a better minimum. This causes the nodes to move where they are needed to resolve the solution.

While the basic idea is simple, there are computational dotails which are essential to make the method work correctly. In regions where the solution is flat, the prescription for moving the nodes becomes indeterminate because a range of different node motions give equally "best" fits. This is manifested mathematically in the vanishing of the determinant of the mass matrix in $\mathrm{E}_{\text {(ls. }}$ (20) and (27), and is resolved by adding regularization terms to $L$ which can be interpreted as internodal viscosity and grid tension. The weight function in $\mathrm{Eq}_{\mathrm{g}}$. (17) is chosen to be $w=\left[1+(\nabla u)^{2}\right]^{-1 / 2}$, which converts the integral over the clomain into an integral over the aren of the solution manifold, resulting in much better node motion and in placing the independent spatial variables $\mathbf{x}$ and the drpendent variables $u$ on more of an equal basis. The time step must be implicit in order to exceed the Courant condition and use efficiently large step sizes. This requires the solution of large, sparse linear systems, which is currently done with a direct band solution but will 
shortly be replaced with a more efficient itcrative method using buffered relaxation as a preconditioner for a nonlinear Krylov subspace method.

In addition to the continuous node motion defined by the MFE method, we use a technique called Graph Massage (GM) to occasionally create and annihilate grid nodes, adding necessary topological flexibility to the continuous grid motion of MFE. Nodes are created when a triangular cell gets too elongated or when the graph gets too jagged (locally nonplanar). Nodes are doleted where they have overaccumulated, without significantly darnaging the graph. For the scrapeoff layer problem, we have modified GM to use an anisotropic metric with principal axes aligned with the mangetic field.

GM has been adapted to the problem of initially triangulating the multiply connected domain of the scrapeoff layer. We start with a crude initial triangulation using only boundary nodes, and then use GM with the anisotropic metric to insert interior nodes. Additional smoothing procedures are used to improve the trinngulation.

\section{Code Results}

A code has been written based on Braginskii's equations, Eqs. (3) - (14), but, this code is not yet working. Our results so far are based on the model tokannak hent equation, Egs. (1) and (2). Our principal diagnostic is a 3D color movie on an SGI Iris workstation. Our figures are stills from that movie. Figures 1 3 show the grid at various stages during the run. The magnetic field is obtained from numerical output from a numerical solution to the Grud-Shafranov equation, with parancters apprpriate to ITER with a single unll divertor. ${ }^{4}$ Our computational domain is bounded by flux surfaces for the core and private flux boundaries and by inboard and outboard divertor plates. Figure 1 shows the initial grid with only boundary nodes. Figure 2 shows the grid after Graph Massnge has been used to generate interior nodes. Figure 3 shows the final grid, containing 1267 nodes, nfter it has been modified by Moving Finite Elements and additional npplications of Graph Massage. Figure 4 shows the temperature distribution at the end of the run.

The status of the code is promising but not yet sutisfuctory. The behavior of Graph Massage is excellent; that of Moving Finite Elements is much less so. After each application of GM, the grid nppenrs well distributed and the solution appenrs well resolved. MFE then moves the grid in such a wny as to make it less woll resolved in some regions, and the extent to which it aligns with the mngnetic field is less than 
expected. We believe this is due primarily to our treatment of gradient weighting. As discussed in Section 3, without gradient weighting the grid has a tendency to overemplasize regions of sharp gradients, leaving inadequate grid to resolve other regions. Gradient weighting reduces this effect when the gradients exceed some critical value determined by input scale factors. For the edge problem, the criterion for gradient weighting should be made anisotropic to properly treat the disparate length scales along and across the mangetic field. Such anisotropic scaling has been incorporated into GM but not MFE. Incorporating it into MFE is our next task.

The speed of the code is also not yet satisfuctory, requiring several hours on an HP 735 workstation. We attribute this to two problems which we hope to solve. The time step depends critically on the success of MFE in moving the grid optimally, and as discussed above this has not yet been acheved. In addition, we use a direct method for large sparse matrix solution on an unstructured grid, and an iterative method could probably improve on this.

\section{Conclusions}

We have described an alternative numerical method for solving the equations of the tokamak scrapeoff layer, using an adaptive grid in a simple coordinnte systom rather than a piecewise uniform grid in a flux coordinate system. We have described a code with a fairly complete set of equations which has becn written but not yet run. We have presented code results for a simpler model problem, showing promising but not yet perfect grid behavior, and we have indicated how this behavior can be perfected.

\section{References}

1. B. Brnums, these procoedings.

2. A. H. Glesser, "A Moving Finite Element Model of the High Density Z-Pinch," Journal of Computational Physics 85, 159 (1989); A. H. Glassor, A. P. Kuprat, and K. Millor, "2D Moving Finite Elemonts: An Adnptive Grid Method for Computational Fluid Dyunuics," tutorial session presented at the Physics Computing '03 Conference, Albuquerque, NM, Mny 31 to Juse 4, 1903.

3. S. I. Brnginskii, Rev. Plasma Physics, edited by M. A. Leontovich (Consultants Bureau, New York, 1985) Vol. I, pl). 2(55-311.

4. L. D. Penrlstein, private communication. 


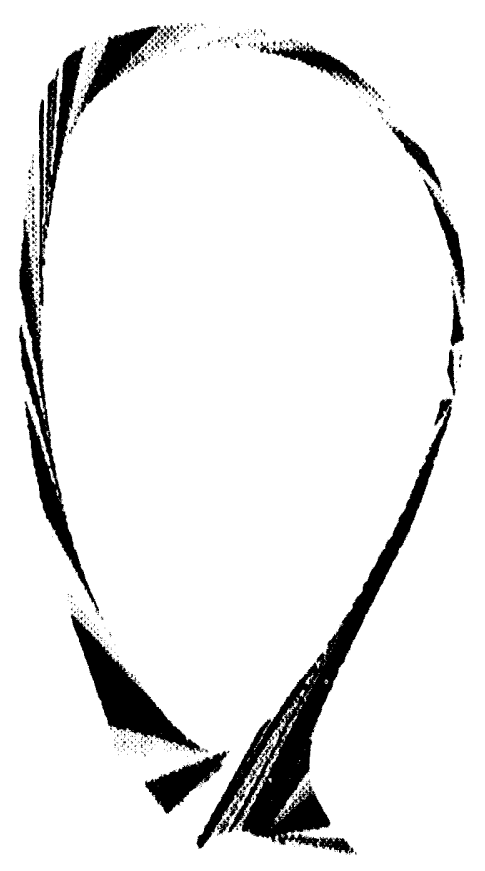

Fig. 1. Initinl grid with only bomblary nodes.

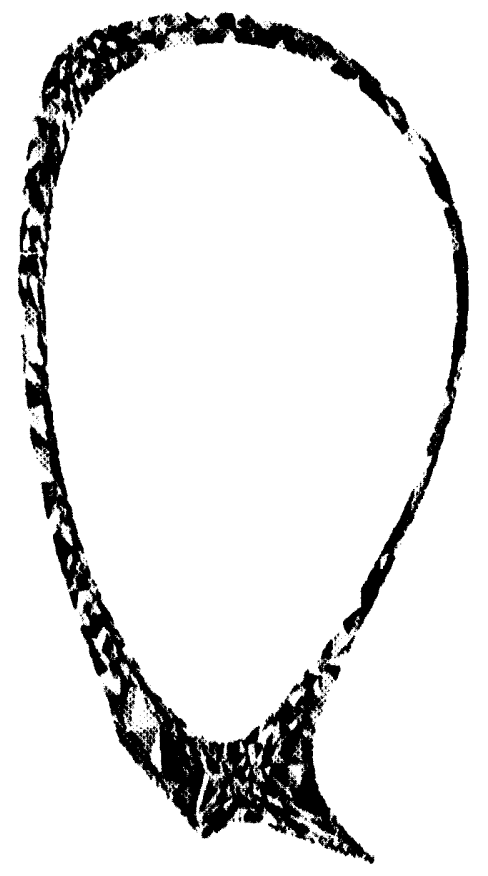

Fig. 3. Finnl gricl after Moving Finite Eloments mal more Girmph Mussnges.

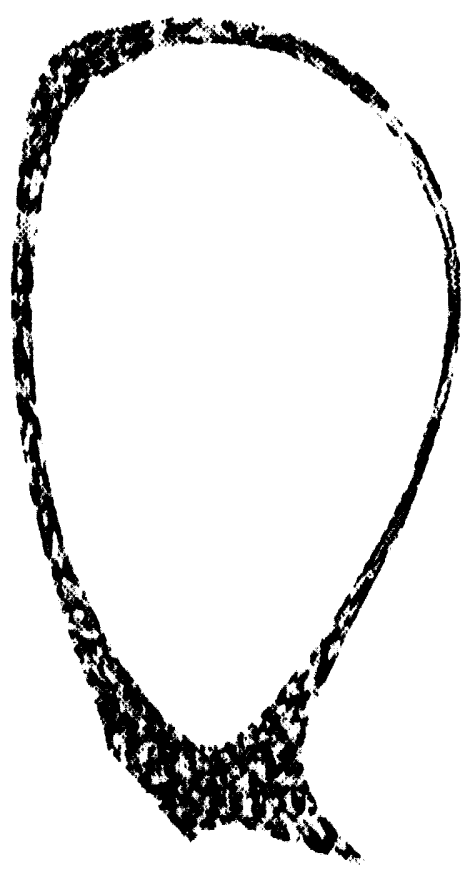

Fig. 2. Initial grid ufter using Cimph Mlassage to generate intermal noders.

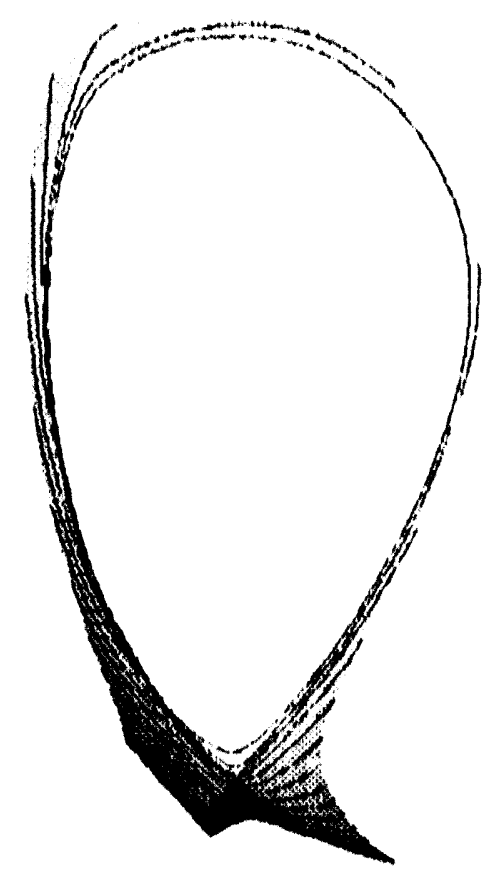

Fig. 4. Final tempernture distribution. 

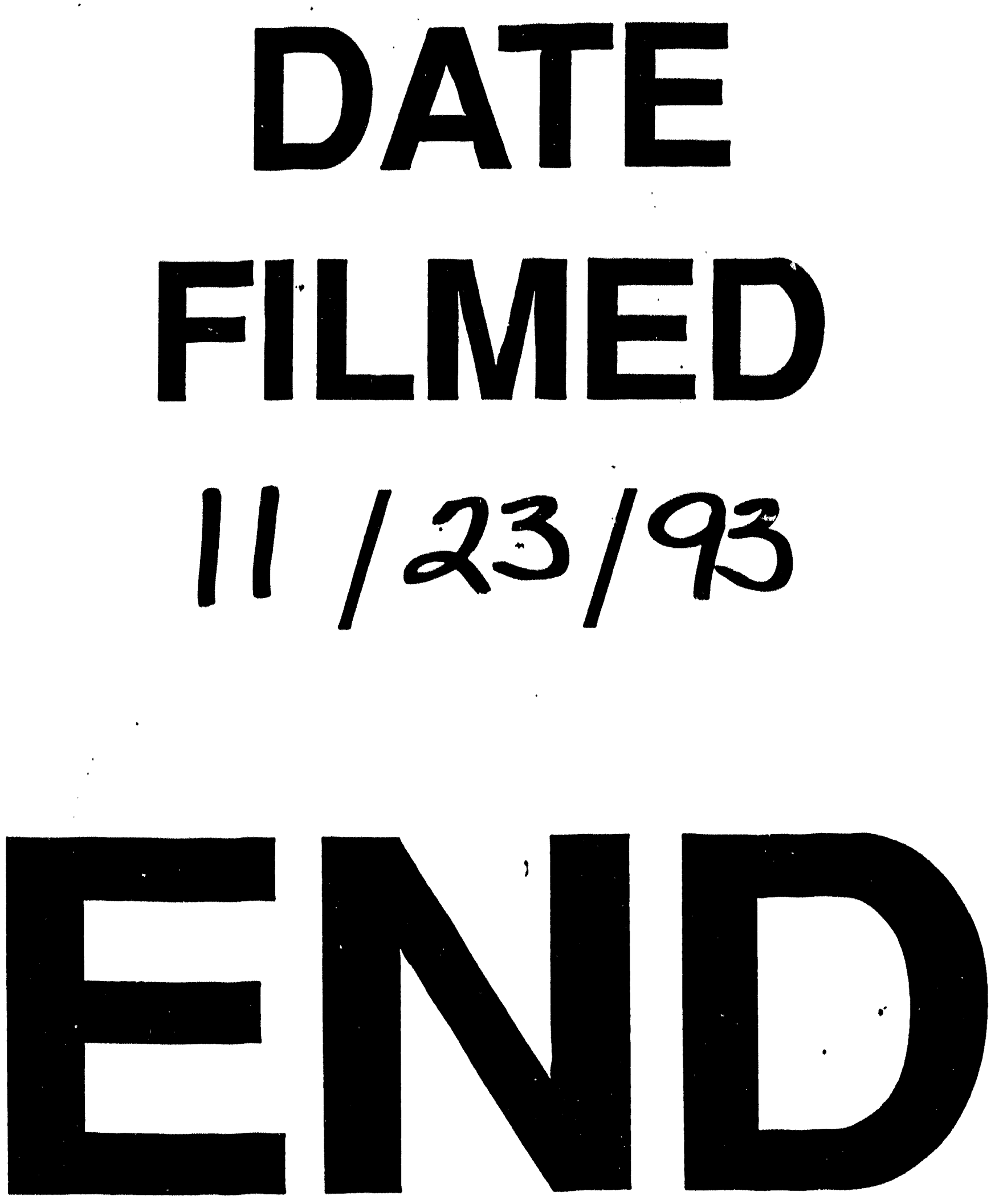